\title{
La interrelación entre las condiciones económicas e institucionales en la regulación de servicios públicos
}

\author{
Silvia Regoli Roa \\ Licenciada en Ciencias Económicas. \\ Prof.Asociado Finanzas y Regulación Dpto. de Economía \\ FCE, UNL.
}

\section{Introducción}

Este artículo analiza la relación entre las condiciones económicas e institucionales de la Regulación de Servicios Públicos. Está basado principalmente en el trabajo del Profesor Jones. ${ }^{(1)}$ Su traducción y análisis se efectuaron por necesidades vinculadas a la docencia de grado y posgrado como a tareas de investigación (tesis de Maestría y Proyecto CAI+D), actividades éstas realizadas por la autora.

El objetivo del trabajo de Jones es aportar al debate actual con relación a los instrumentos de regulación. Su trabajo representa un intento de identificar las instituciones regulatorias apropiadas para las condiciones que presentan economías con un grado de desarrollo bajo e intermedio, las que enfrentan escasez de capital humano calificado en temas de regulación y marcados déficit institucionales y en las cuales el poder de mercado que tienen las empresas privadas es elevado. Vale decir que procura identificar la tecnología adecuada a la dotación de recursos que caracteriza estas economías.
Se sabe que una apropiada regulación significa maximizar los beneficios derivados de remover las fallas de mercado con relación a los costos de la intervención pública y que los mercados monopólicos fallan debido a ineficiencias productivas y en la asignación de recursos, principalmente. Las regulaciones que mitigan las ineficiencias asignativas pero exacerban las ineficiencias de costos son inapropiadas porque los costos de la intervención sobrepasan los beneficios.

Jones arriba a la interesante conclusión de que los beneficios potenciales de la regulación son mayores en los países menos desarrollados, dado que el efecto conjunto de las fallas de mercado es más grande que en los países desarrollados.

Aunque alerta sobre el hecho de que también son elevados los costos de intervención debido a las fallas del Estado, que son mayores en este grupo de países que en los desarrollados. Por lo que en cada caso deben sopesarse ambos tipos de fallas.

(1) Jones, L. Appropiate regulatory technology. The interplay of economic and institucional conditions, Proceedings of the World Bank Annual Conference on Development Economics, BIRF, 1994. 
La intervención debe procurar controlar las ineficiencias asignativas a través de mecanismos de bajo costo. Los métodos de Chile y Nueva Zelanda representan diferentes formas de hacer esto y proveen modelos apropiados para países poco desarrollados.

\section{Beneficios y costos de la regulación}

Los beneficios de la regulación pueden pensarse como las ganancias de bienestar derivadas de la corrección de las fallas de mercado en orden a alcanzar un resultado Pareto-eficiente.

Los costos de la regulación son aquellos derivados de las fallas del Estado para rectificar los problemas anteriores sin introducir distorsiones adicionales.

Los costos incluyen los costos de oportunidad de los recursos públicos y privados dedicados al proceso de regulación, así como también la posibilidad de que instituciones imperfectas operando con información imperfecta puedan crear nuevas distorsiones en su intento muchas veces bienintencionado de reducirlas o eliminarlas.

De igual manera, las fallas de mercado no sólo incluyen los problemas creados por el poder de mercado sino también los asociados a problemas de agencia, costos de transacción, de información, contratos imperfectos o incompletos, etc.

Para Jones, existe una gran variación en la magnitud y signo de los costos en diferentes entornos institucionales.

Una regulación se diseña para maximizar los beneficios netos para una industria país y tiempo determinados, vale decir es una regulación situada, a medida. En esto es coincidente con la opinión de otros autores como Sparrow quien se refiere al proceso de regulación como a una tarea artesanal. (2)
Ambos autores consideran que se debe procurar estimar en cada caso particular cuál es la magnitud de los costos y beneficios de la regulación.

Jones analiza los argumentos que se mencionan en la literatura en contra de la regulación.

Para quienes plantean que la alternativa es no regular, el argumento más común se basa en que los costos de la regulación exceden los beneficios. Jones presenta un cálculo de tales distorsiones basándose en estimaciones realizadas para EE.UU.

Analiza luego la forma en que se pueden medir los costos de la regulación y señala que esto depende del mecanismo institucional que se elija. Arriba a la conclusión de que los costos de oportunidad son considerables y serán mayores en países poco desarrollados en los que los recursos humanos con las calificaciones requeridas para regular son escasos.

№ hay razón para que las distorsiones de precios y costos sean de igual magnitud pero la cuestión es que la distorsión de costos es mucho más importante y aún una pequeña ineficiencia de costos puede superar las ganancias de un recorte de precios considerable.

La primera falla del argumento de no regulación es que se apoya en distorsiones de precios relativamente modestas. Para una economía industrializada, este es un supuesto plausible ya que muchos sectores no tendrán grandes distorsiones y otras ya habrán sido reducidas por la regulación.

$\mathrm{Si}$, en cambio, se estiman los beneficios potenciales de pasar de cero a una regulación perfecta en un sector particular, la magnitud de la distorsión puede ser considerablemente grande.

Hay una relación inversa entre la elasticidad, precio de la demanda y la distorsión del precio óptimo. Cuanto menor es la elasticidad, mayor será el aumento de precios que fije un monopolista maximizador. Luego, bajas elasticidades están acompañadas de mayores distorsiones de precios. Las bajas elasticidades generan mayores pérdidas de asignación debido al monopolio, y viceversa.

(2) Sparrow, M. The regulatory craft, controlling risks, solving problems and managing compliance, Brookings Institution, Washington, 2000. 
En consecuencia, la sociedad tiene poco que temer a monopolios que enfrenten una elasticidad alta y mucho que temer a los monopolios que enfrenten una demanda inelástica, tal el caso de los Servicios Públicos. La elasticidad de demanda varía considerablemente entre sectores, países, métodos de estimación pero es claro que en algunas industrias clave, el valor de la elasticidad es bastante bajo. En electricidad, la elasticidad de la demanda a corto plazo es muy baja (próximo a 0,2) y la pérdida de bienestar asociada sería muy grande (62,5\% del ingreso).

Las elasticidades de largo plazo son siempre mayores porque la gente tiene tiempo de adaptar su comportamiento. Una elasticidad de corto plazo de 0.2 puede terminar en una elasticidad de largo plazo de 2 y la pérdida de bienestar asociada sería $6 \%$ solamente. Entonces, icuál es realmente la magnitud de la pérdida de bienestar $62 \% 06 \%$ ?

Será un promedio ponderado donde las ponderaciones están en función de la tasa de descuento del monopolista. El monopolista maximizará el valor presente de su corriente de ingreso. Dada la elevada tasa de descuento para el capital empresarial en los países poco desarrollados (alrededor del $30 \%$ ), los primeros años pesan más en la decisión y el promedio ser más cercano a $60 \%$ que a $6 \%$.

En resumen, las ineficiencias asignativas no siempre son pequeñas y el campo para las mejoras en la asignación de recursos derivadas de la regulación es sustancial, especialmente en los países poco desarrollados.

En los sectores de Servicios Públicos es posible obtener beneficios netos sustanciales de la regulación siempre que se eviten mecanismos que induzcan ineficiencias en los costos u otras distorsiones.

Jones analiza qué aspectos serán diferentes en los países poco desarrollados respecto de los países desarrollados. Uno de los aspectos diferenciales es la concentración industrial.

La concentración de una industria depende de la relación entre el tamaño de planta óptimo y el ta- maño del mercado. Los países poco desarrollados tienen mercados más pequeños. Si la tecnología estuviera estandarizada, todos los países tendrían igual tecnología y, por tanto, el mismo tamaño de planta óptimo o eficiente. Pero las economías más desarrolladas y prósperas podrán alojar mayor número de empresas que las economías menos desarrolladas. Esto implica que las últimas tendrán una mayor concentración que las primeras.

Las tecnologías se ajustan pero no totalmente, máxime en un mundo tan globalizado. La elasticidad del tamaño de planta respecto del tamaño de mercado es aproximadamente 0,5 según estimaciones de Caves (1989).

Si el tamaño de planta declina menos rápidamente que el tamaño del mercado, los estudios empíricos muestran un aumento en la concentración a medida que decrece el tamaño de la economía (Scherer y Ross, 1990).

Otros factores que afectan la concentración son el grado de apertura comercial, la calidad del sistema de transporte, etc. (Lee, 1984). En muchos países de bajo desarrollo, los sectores modernos son más concentrados pero también tienen menor participación en el PBI.

El segundo problema con el argumento de no regulación es que ignora las fallas originadas por la búsqueda de rentas (rent seeking).

Un problema diferente con el argumento de no regulación es que ignora la realidad política de las presiones populares (sindicatos y otros grupos de interés) para regular. Estos argumentos son especialmente importantes en los países poco desarrollados, muchas veces asociados a la equidad distributiva. Pero este debe ser analizado con cuidado. Por ejemplo, en muchos países la gente realmente pobre no tiene acceso a los servicios públicos esenciales, especialmente en las zonas alejadas y rurales como es el caso del agua potable, el transporte público o el gas natural.

Aquellos grupos que están comparativamente mejor tienen acceso a todos los servicios a tarifas relativamente bajas que muchas veces no al- 
canzan para encarar ampliaciones a la red. Las bajas tarifas benefician a sectores de ingresos medios y medio altos y perjudican a grupos de ingresos bajos, que deben consumir sustitutos a precios elevados.

Si los mayores ingresos por mayores tarifas fueran apropiados por el Estado a través de impuestos a las privatizadas 0 a través de las empresas públicas podrían volcarse en subsidios y demás instrumento de redistribución de ingresos hacia los grupos más carenciados.

Sin embargo, la redistribución se da principalmente desde los grupos de medianos ingresos hacia las empresas privatizadas.

Los políticos, ante la dificultad de alcanzar posiciones Pareto-eficientes optan por lo que Jones denomina posiciones político-eficientes, es decir aquellas donde alguien mejora su posición sin que otros se den cuenta de que ha empeorado la suya. Muchas reasignaciones Pareto-ineficientes pueden ser Político-eficientes, y muchos políticos optan por regular para bien o para mal.

En resumen, el argumento de no regulación es algo retórico establecido para confirmar estas cuestiones:

1) Los beneficios de la regulación se sobreestiman fácilmente

2) Los costos de la regulación no son reconocidos, incluyen costos en recursos y grandes distorsiones debidas potencialmente a la regulación

3) Los beneficios netos de la regulación pueden ser fácilmente negativos

4) Existe un campo para la regulación que mejora el bienestar pero los objetivos deben ser cuidadosamente seleccionados y los mecanismos deben ser diseñados de modo de minimizar los costos con relación a los beneficios

5) Se debe prestar especial atención a las ineficiencias de costos. Esto implica establecer los beneficios derivados de remover las fallas de mercado y evitar los costos asociados a las fallas del Estado.

\section{Regulación de precios}

La tecnología regulatoria se caracteriza por la disminución de los beneficios marginales y el incremento de los costos marginales. Los beneficios marginales de remover las ineficiencias asignativas son decrecientes. Esto significa que eliminar el primer $1 \%$ de una distorsión asignativa de un $20 \%$ es mucho más beneficioso que remover el último 1\% (suponiendo una elasticidad unitaria). Los costos aumentan por las dificultades y los costos de obtener la información.

No es difícil, por ejemplo, determinar si los precios de una empresa están sobre el nivel de eficiencia ya que las comparaciones internacionales arrojan mucha información relevante. Pero demostrar que una empresa está un pequeño porcentaje por encima del nivel de eficiencia sería tremendamente costoso y quizás imposible.

La lección es: se debe tratar de controlar los problemas de eficiencia más serios y dejar el resto.

Los distintos mecanismos de regulación pueden ser evaluados según estos criterios.

El mark-up o margen de rentabilidad sobre todos los costos y la tasa de retorno sobre el capital requieren abundante información y provocan ineficiencias productivas. La empresa no tiene incentivos a ahorrar costos sino todo lo contrario.

Se han propuesto varios mecanismos para minimizar este problema. Laffont y Tirole (1993) señalan que estos esquemas varían según el porcentaje que se le deja a la empresa con relación a su ahorro de costos.

El price cap fijo es el más poderoso como incentivo al ahorro de costos. Pero este esquema plantea un trade off entre equidad y eficiencia.

Con un retraso regulatorio finito, la empresa sabe que cuando se fije el próximo precio el menor costo será tenido en cuenta, y los consumidores o el gobierno se apropiarán de parte de tal reducción, lo que disminuye el incentivo al ahorro de costos.

El price cap tal como se lo aplica en Gran Bretaña, es un instrumento de regulación interesante. Una ventaja adicional es el rezago ya que ajusta 
explícitamente por una de las variables más exógenas: la inflación. Dadas las altas tasas de inflación en los países de menor desarrollo éste no es un incentivo menor.

En suma, el incentivo de un sistema de regulación de precios no depende tanto del esquema específico sino del rezago regulatorio y de las expectativas de cómo se ajustarán los precios al final del período.

Al price-cap se lo asocia con una mayor simplicidad en su implementación. Así es en Nueva Zelanda donde al sistema se lo llama "regulación sin reguladores". No hay cuerpos específicos de regulación para cada industria y las restricciones están contenidas en las regulaciones. En el caso de teléfonos, éstas establecen que la renta de una línea residencial estándar no puede crecer más que la variación en el Índice de precios al consumidor (a menos que el operador local presente un desequilibrio no razonable), y que las tarifas rurales no pueden crecer más que las residenciales. Los precios de los demás servicios los regula la competencia o el temor a la misma.

Un gerente cauteloso evitaría que los beneficios aumenten en forma irrazonable, idealmente podría bajar los precios pero también podría no aprovechar todas las oportunidades para bajar costos. No ataca el segundo mal del monopolio dejando el problema de la gobernanza corporativa al sector privado.

El primer énfasis es en los incentivos de eficiencia productiva, bajar costos, con controles más débiles sobre la ineficiencia asignativa, es decir, sobre la fijación de precios del monopolista.

Chile emplea un modelo distinto que puede ser descrito como cost plus fair return ya que a la empresa se le permite una tasa de retorno igual a una tasa libre de riesgo y la rentabilidad de un conjunto diversificado de inversiones.

Se ajusta cada dos meses para reflejar la inflación. La diferencia real con el price cap reside, primero, en el retraso, a que en Chile el período de ajuste es explícito y refleja un largo rezago de 5 años. Adicionalmente a la tasa de retorno razona- ble se calculan los costos marginales a largo plazo en el contexto de un plan de inversiones con un horizonte temporal de 5 años (diseñado para satisfacer la demanda proyectada), el mark-up (o diferencia) entre costo marginal y medio se distribuye en base a precios Ramsey y así siguiendo a esta altura parece claro el nivel de sofisticación del instrumento utilizado en Chile.

Este mecanismo reduce la incertidumbre de la empresa y focaliza la discusión en los parámetros tecnológicos. La diferencia con el sistema de USA radica en la especificidad ex ante respecto de la forma de determinar los parámetros. En Chile los principios se detallan con anterioridad.

El segundo punto clave es que el modelo se basa también en datos de empresas de otros países. Este precio por comparación es incuestionable. Dada una cantidad suficientemente grande de empresas se pueden usar métodos econométricos para establecer la frontera tecnológica óptima (Benchmarking). Adicionándole los datos sobre los precios internos y el costo del capital es posible generar el precio correcto para la empresa a regular. El problema es que no hay un número suficientemente grande de empresas y el ajuste debe hacerse para aquellas que se regulan en el país.

En el caso del sector eléctrico este problema se minimiza por la existencia de un número considerable de empresas de distribución similares. Se recopilan los datos para el conjunto y se establece la comparación a nivel local (Chile). Forzada a alcanzar la frontera tecnológica una empresa no tendrá incentivo de desviarse ya que sus propios costos son una pequeña parte del total.

\section{Sub inversión: expropiación vs. explotación}

Si existe temor por parte de la empresa de que sus ganancias futuras serán expropiadas por el regulador, esto puede inducir a la subinversión. 
Éste es un problema muy serio en contextos monopólicos y la ampliación de la inversión es uno de los objetivos de la regulación.

La subinversión puede deberse a varias causas, no sólo al temor a la expropiación de las ganancias. Otra causa es el temor al rescate anticipado una vez realizadas las inversiones 0 simplemente a un contexto regulatorio excesivamente amigable, en el que la empresa explotará su poder monopólico creando un exceso de demanda y logrando incrementos de tarifas. Las mayores rentas finalmente irán a incrementar costos no a ampliar el servicio.

Esto puede ocurrir aun cuando las empresas de servicios públicos no estén en manos privadas. El financiamiento insuficiente 0 la búsqueda de rentas puede conducir a la subinversión, dando lugar al racionamiento de los servicios mediante la cola, llegando a ser los tiempos de espera excesivamente largos, 0 bien conducirá al surgimiento de un mercado paralelo donde los usuarios que deseen el servicio deberán pagar ingentes sumas de dinero a personas con conexiones dentro de las empresas. Estos pagos pueden beneficiar a quienes los reciben pero con seguridad no serán destinados a mayor inversión.

Es de gran importancia para mitigar la subinversión, la credibilidad de que goce el marco regulatorio y la forma en que se conducirán en el futuro el regulador y el poder concedente. Igualmente importante es la transparencia de las reglas de fijación de precios, calidad y de las inversiones exigidas en el contrato.

Laffont y Tirole (1993) sugieren entre otros mecanismos reembolsar una porción importante de las inversiones, o permitir a la empresa cuando sea posible usar el capital instalado para producir bienes para mercados no regulados.

\section{A modo de conclusión}

La literatura analizada destaca el hecho de que en los países con menor grado de desarrollo, los mercados están más distorsionados que en los desarrollados. Esto se debe a que el déficit de capacidad institucional y las diferencias en la dotación de factores torna más difícil enfrentar los problemas derivados del poder de mercado.

El diseño de la regulación debe maximizar los beneficios netos de la misma para una industria, país y tiempo determinados y concentrarse en promover los incentivos adecuados al ahorro de costos por parte de las empresas reguladas mediante instrumentos simples y de bajo costo. En vista de la escasez de instrumentos para resolver los problemas en los países menos desarrollados, éstos deben dirigirse a solucionar los abusos más serios, lo que implica atacar principalmente las ineficiencias productivas más que las asignativas.

Jones concuerda en que la regulación debe impedir conductas de explotación o expropiación de la empresa por parte del gobierno u otros grupos de presión, pero destaca la importancia de dedicar igual o mayor atención a evitar la explotación de los usuarios por parte de la empresa regulada y de tener en cuenta a los futuros usuarios garantizando una expansión adecuada de las inversiones.

Resulta clara la importancia que dan todos los autores mencionados, y Jones en particular, a la interrelación entre las condiciones económicas e institucionales de la regulación. Considero que esto debe tenerse en cuenta a efectos de no encerrarse en análisis exclusivamente mono-disciplinarios. 
Regoli Roa / La interrelación entre las condiciones económicas e institucionales...

\section{Bibliografía}

- Caves, R. "International Differences in Industrial Organization", en Handbook of

- Lee, N. Business "Concentration in Developing Countries" en Kirpatrick, Industrial Organization, Schmalensee, R. Ch. et al., Industrial Structure and PoyWillig, R. North-Holland: New York, 1989. licy in Less Developed Countries, Allen - Jones, "L. Appropiate regulatory technology. The interplay of economic and institucional conditions, Proceedings of the World Bank Annual Conference on Development Economics," BIRF, 1994. - Laffont, J.J. y Tirole, J. A theorty of Incentives in Procurement and Regulation. MIT: Cambridge, 1993. and Unwin: London, 1984.

-Scherer, F.M. y Ross, D. Industrial Markets and Economic Performance. Houghton Mifflin, Boston, 1990.

-Sparrow, M. The regulatory craft, controlling risks, solving problems and managing compliance, Brookings Institution, Washington, 2000. 